\title{
Linx
}

Revue des linguistes de l'université Paris X Nanterre

$7 \mid 1995$

Saussure aujourd'hui

\section{Jakobson sous le pavillon saussurien}

\section{Françoise Gadet}

\section{OpenEdition}

Journals

Édition électronique

URL : http://journals.openedition.org/linx/1238

DOI : 10.4000/linx.1238

ISSN : 2118-9692

\section{Éditeur}

Presses universitaires de Paris Nanterre

\section{Édition imprimée}

Date de publication : 1 septembre 1995

Pagination : 449-459

ISSN : 0246-8743

\section{Référence électronique}

Françoise Gadet, « Jakobson sous le pavillon saussurien », Linx [En ligne], 7| 1995, mis en ligne le 24 juillet 2012, consulté le 10 décembre 2020. URL : http://journals.openedition.org/linx/1238 ; DOI https://doi.org/10.4000/linx.1238

Ce document a été généré automatiquement le 10 décembre 2020.

Département de Sciences du langage, Université Paris Ouest 


\title{
Jakobson sous le pavillon saussurien
}

\author{
Françoise Gadet
}

1 Contrairement à d'autres linguistes «structuralistes », Roman Jakobson ne se déclare guère lui-même comme héritier de Saussure, et encore moins l'unique ou le véritable héritier de Saussure. Saussure est pour lui une source d'inspiration parmi d'autres (et sûrement pas la plus importante), qui sont: l'Ecole de Kazan, autour de Baudouin de Courtenay et Kruszewski; les Formalistes, les Futuristes et l'Avant-garde russes; Brentano, Anton Marty, Husserl, la Gestalt, Bühler; et, à partir des années cinquante, Peirce ou la théorie de l'information... Toutefois, les références à Saussure sont très nombreuses ${ }^{1}$ tout au long de sa carrière, même si elles apparaissent souvent critiques.

2 Nous centrerons notre étude sur une période allant de l'arrivée de Jakobson à Prague (1920) à son arrivée à New York et l'enseignement d'un cours à l'Ecole Libre des Hautes Etudes (1942), en nous arrêtant à l'épisode crucial de l'élaboration des Thèses de Prague (1929) ; période décisive de sa maturité scientifique (entre 25 et 45 ans), au terme de laquelle il rencontre Claude Lévi-Strauss (1942), à qui il fait connaître Saussure qui deviendra pour lui aussi une référence essentielle, au point que, revenu en France, il le fera à son tour connaître à Jacques Lacan, au début des années cinquante.

\section{Jakobson et Troubetzkoy}

3 Pour la période entre les deux guerres, on ne peut parler de Jakobson sans évoquer le Prince Nicolas Troubetzkoy, car la fin des années vingt et le début des années trente voient une collaboration étroite entre les deux hommes, matérialisée pour nous par leur correspondance, dont seules les lettres de Troubetzkoy sont conservées.

Il y a pourtant, au moins sur la relation à Saussure, une nette différence entre eux. Troubetzkoy s'irritait qu'on le donne comme disciple de Saussure. Il a connu Saussure par Jakobson, tardivement dans une carrière à la fois précoce et brève, et la première mention qu'il en fait, en 1923, concerne le Mémoire; quant au CLG, il l'a peu lu². Manque d'intérêt que, dans sa nécrologie de Troubetzkoy (1939, in 1971b, p. 502 sq), Jakobson explique par le fait que c'était un savant « durch und durch historisch eingestellt », que 
les problèmes de synchronie laissaient «kühl und passiv». L'ensemble de l'œuvre publique ne comporte guère qu'une dizaine de mentions, la plupart critiques. Elles sont toutefois plus nombreuses dans la correspondance, mais encore plus critiques.

5 La première référence publique au CLG (et la plus développée) apparaît en 1933, lors d'une tentative pour présenter « la phonologie actuelle » en la différenciant de l'orientation des prédécesseurs. La critique à Saussure porte sur le problème du changement, dont l'explication par la seule logique interne de la langue ne permet pas, selon Troubetzkoy, d'expliquer la cause première. Il reproche à la conception saussurienne, par « concession à l'atomisme des Néo-grammairiens ", de conduire à concevoir le changement comme intervenant sur des faits isolés, ce qui introduit du désordre dans le système. Car «si, à chaque moment donné, la langue est un 'système où tout se tient', le passage d'un état de langue à un autre ne peut être effectué par des changements isolés dénués de tout sens ». Si l'on veut pouvoir comprendre l'évolution, il faut admettre que les changements ont une raison d'être, une téléologie, il faut considérer que «l'évolution du système phonologique est à chaque moment donné dirigée par la tendance vers un but ».

La seule autre mention publique, au début des Grundzüge der Phonologie (parus en 1939), concerne langue/parole et signifiant / signifié ; ce texte, le plus connu de Troubetzkoy, a imposé une image amplifiée de l'importance que l'œuvre de Saussure avait pu avoir pour lui.

\section{Jakobson jusqu'en 1939}

Il en va différemment de Jakobson, qui a été l'un des premiers à citer Saussure dans une langue autre que le français. Il le connaît dès l'époque de Moscou, par le truchement oral de Serge Karcevski ${ }^{3}$, qui circule entre Genève et Moscou. Albert Séchehaye lui a envoyé dès 1920 un exemplaire du CLG, mais on ne sait s'il l'a lu tout de suite. Toujours est-il qu'entre 1921 et 1939, on peut relever trois fois plus de mentions de Saussure dans les publications de Jakobson que dans celles de Troubetzkoy (Viel, 1984, p. 57), et, si beaucoup d'entre elles sont critiques quant aux dichotomies, les hommages ne manquent pas sur la conception globale.

Nous ne ferons ici qu'évoquer quelques points de repère, à partir de la première mention dans la Nouvelle poésie russe, texte paru en 1921 mais écrit en 1919 à Moscou, dans lequel c'est à Saussure que Jakobson emprunte son appareil conceptuel ${ }^{4}$, langue/parole et surtout synchronie/diachronie. Même si dès ce moment il propose de "dépasser " ${ }^{5}$ cette dernière antinomie, la tonalité d'ensemble parait plutôt d'adhésion au modèle saussurien.

Synchronie/diachronie est donc à la fois le premier couple conceptuel saussurien mis en application par Jakobson, et le premier soumis à la critique : dès la fin de 1926, Jakobson parle dans une lettre à Troubetzkoy de « combler le fossé contre-nature entre l'analyse synchronique et la phonétique historique en considérant chaque changement phonétique du point de vue du système global » (1975).

L'évolution vers une attitude plus critique intervient dans la période qui suit la création en 1926 du Cercle de Prague, lors de laquelle sont rédigés à la fois "O hlaskoslovném zakonu a teleologickém hlaskoslovi » (1928, repris en traduction anglaise in 1971a, «The Concept of the Sound Law and the Teleological Criterion») et de la "Proposition " présentée en 1927 pour le Congrès de la Haye (1928, in 1971a), en réponse à la question soumise "Quelles sont les méthodes les mieux appropriées à un exposé complet et 
pratique de la grammaire d'une langue quelconque ?»; la proposition, qui ne répond que pour la phonologie, est rédigée par le seul Jakobson, et contresignée par Troubetzkoy et Karcevski ${ }^{6}$.

11 Les argumentations des deux textes étant très proches, nous ne parlerons que de la Proposition, un peu plus développée. Parmi les points qu'elle présente, c'est surtout le deuxième qui concerne Saussure, bien qu'encore une fois le texte s'ouvre sur l'évocation de son nom?

Décrire la phonologie d'une langue impose avant tout de tenir compte du rôle des sons dans le système phonologique, non seulement en en dressant le répertoire (comme le proposait le $C L G$ ), mais en évitant de ne les caractériser que du point de vue de la production, sans préciser leur rôle dans le système. Il faut aussi «spécifier les types de différences phonologiques significatives ».

Si l'on admet pour ce premier point, en synchronie, que l'on ait dû mettre en avant une perspective fonctionnelle, il faut faire de même en diachronie, et, en supprimant "l'antinomie de la phonologie synchronique et de la phonétique diachronique", " abandonner l'ornière des Junggrammatiker ». Le changement constitue donc le point crucial d'opposition à Saussure: "La conception selon laquelle les changements phonétiques sont fortuits et involontaires et que la langue ne prémédite rien nous nous faisait représenter [sic] la phonétique historique d'une langue comme une suite de troubles et de destructions aveugles causés par des facteurs extrinsèques du point de vue du système phonologique ». Une telle conception ne laisse aux locuteurs que la possibilité de "trouver un sens à l'état de désordre ", mais leur rôle est en fait plus actif, comme le montrent les réflexions que soulève le réexamen de la métaphore du jeu d'échecs, à laquelle Saussure voyait une limite ("Il n'y a qu'un point où la comparaison soit en défaut : le joueur d'échecs a l'intention d'opérer le déplacement », CLG p. 127). Jakobson, quant à lui, n'en voit pas : « L'analogie saussurienne entre la langue et le jeu d'échecs peut être poussée jusqu'au bout. Il existe des changements linguistiques qui [...] ont l'intention d'exercer une action sur le système ».

La conclusion est programmatique: en conséquence de cette mise au premier plan du "problème des tendances et des buts des faits phonétiques », il faut donner la priorité au point de vue acoustique. Jakobson retrouve ici Saussure en proclamant cette priorité, qu'il n'exploite à ce moment pas plus que ne l'a fait Saussure; c'est cependant un thème sur lequel il reviendra avec la formulation des traits en termes acoustiques, surtout à partir de $1938^{8}$.

15 Ce texte de Jakobson reflète des thèmes qui sont les siens tout au long des années vingt, et qui affleurent aussi dans les Thèses de 1929, où ils sont toutefois exprimés sous une forme moins polémique, peut-être par un effet de la rédaction multiple. Le nom de Saussure n'y apparait pas (ne figure d'ailleurs aucun nom propre), mais les concepts discutés, surtout synchronie/diachronie, sont en grande partie ceux de "l'Ecole de Genève ", et c'est là aussi au nom du fonctionnalisme et de la téléologie qu'ils sont mis en cause.

16 Au cours des années trente, les mentions de Saussure sont moins nombreuses, et Waugh (1984), qui présente un relevé des principaux articles discutant les thèses saussuriennes, n'en signale, après la riche période de la conception des Thèses (quatre articles en 1928 et 1929, sans compter les Thèses elles-mêmes), que cinq entre 1930 et 1939 (tous repris in $1971 b$; encore la discussion des concepts saussuriens n'y est-elle pas centrale). 
17 C'est donc en cette première période surtout sur langue/parole et synchronie/diachronie qu'est ciblée la critique, bien qu'aux alentours de 1939 apparaissent les premières discussions sur l'arbitraire du signe (Communication au 5ème Congrès des Linguistes de Bruxelles, 1939), sur la linéarité du signifiant («Zur Struktur des Phonems », 1939, in 1971a), et sur le signe zéro (« Signe zéro », 1939, in 1971b, et « das Nullzeichen », 1939, in 1971b). Mais alors que la critique de langue/parole se fait dans un sens de réflexion et d'analyse, celle de synchronie/diachronie est moins respectueuse de la lettre du texte saussurien, en figeant une dichotomie qui n'est pas une exclusive dans le CLG (cf. Fontaine, 1974).

18 L'attitude élaborée au cours de cette période ne se modifiera fondamentalement pas par la suite : très imprégnée du CLG, mais polémique à son égard, au point que Engler (1990) a pu parler d'un « atteggiamento contraddittorio ", à la fois une « adesione implicita, e una critica quasi totale ", ce qui, dans les années trente, le distingue assez nettement de l'attitude des autres linguistes européens, qui sont soit acquis, soit hostiles.

\section{Jakobson en 1942}

Jakobson n'a jamais publié ce texte d'un cours professé en 1942 à l'Ecole Libre des Hautes Etudes à New York. Enseignant pour la première fois en français, peu après son arrivée aux Etats-Unis, il rédige tout, du moins au début. Mais alors qu'il laissera publier en 1976 Six leçons sur le son et le sens, enseignement dispensé en parallèle au cours sur Saussure, il ne publiera pas ces notes, que Linda Waugh a retrouvées dans les papiers désormais déposés à MIT Library. Le seul passage détaillé concerne langue/parole, et représente le raisonnement le plus argumenté dont nous disposions de sa part sur cette dichotomie.

Dans une assez longue introduction historique, il commence par l'un des hommages les plus appuyés qu'il ait jusque là accordé à Saussure : le CLG, qui procède non selon des " dogmes définitifs ", mais par des " hypothèses de travail et des esquisses lucides ", n'est pas exempt de contradictions («une œuvre géniale dont même les erreurs et les contradictions sont évocatrices »), mais constitue un point de départ permettant de voir « in statu nascendi [...] presque tous les problèmes essentiels de la pensée linguistique moderne ». Il le trouve toutefois excessivement atomiste et réducteur.

21 Il suit la démarche du CLG en partant de la caractérisation du langage que Saussure définit comme «amas confus de choses hétéroclites sans lien entre elles» (p. 24). Jakobson refuse ce point de départ : on ne peut dire cela qu'en adoptant une démarche atomiste qui ne prend pas en compte la fonction (« ce qui importe avant tout dans tout outil [...], c'est sa fonction »). On ne peut isoler ni le son, ni l'idée, et c'est bien « l'observateur soi-disant superficiel du langage qui a raison » en considérant que le mot est l'expression d'une idée.

Il faut rejeter la conception à l'œuvre tout au long du XIXè siècle, selon laquelle c'est le langage individuel qui représente l'unique réalité linguistique. La notion saussurienne de langue, définie comme "ensemble de conventions nécessaires, adoptées par le corps social pour permettre l'exercice de la faculté du langage chez les individus », permet de rééquilibrer le point de vue. La dichotomie langue/parole lui semble donc justifiée, à condition de la réviser, car elle recèle plusieurs oppositions, qui ne se recouvrent pas toujours.

23 Langue/parole contient à la fois une opposition entre " virtualité » et « actualisation », et entre «collectif» et « personnel». Mais ces deux couples se recouvrent-ils ? Pas le moins 
du monde. La langue et la parole ont à la fois un côté social et un côté individuel, et en assimilant " parole » et « individuel », en affirmant qu'il n'y a « rien de collectif dans la parole ", Saussure néglige le rôle de l'auditeur, et fait fi de sa propre élaboration du circuit de la parole. En fait, la parole est un phénomène intersubjectif : « Le destinateur et le destinataire se supposent nécessairement et la parole porte dans sa structure l'empreinte de tous les deux ». L'évocation du dialogue, de la parole intérieure, du langage enfantin et de la fonction expressive le conduit à conclure : «La langue a à côté de l'aspect social un aspect individuel et la parole a à côté de l'aspect individuel un aspect social».

L'introduction d'un point de vue fonctionnel renforce l'interrogation sur les différentes oppositions, car la fonction émotive ne peut s'identifier avec la couche individuelle du langage. Une autre opposition saussurienne doit intervenir ici, celle entre "esprit particulariste " et "conformisme ", qui lui semble s'appliquer à la fois à langue et à parole, dans leurs aspects subjectifs et intersubjectifs, à condition de l'étendre, du domaine spatial où la fait intervenir Saussure, au domaine temporel.

En exposant la solidarité de l'antinomie continuité/altération, Saussure a le tort de chercher à la ramener à langue/parole, avec l'idée que tout ce qui est mobile ne l'est que par la parole («C'est la parole qui fait évoluer la langue», CLG, p. 37). Mais si toute création est précédée d'une comparaison inconsciente des matériaux qu'offre la langue, et si les éléments de la création sont déjà présents en puissance (ce que dit le CLG), alors c'est bien dans la langue et non dans la parole qu'il faut chercher le germe des innovations, et Saussure se contredit lui-même. L'argument contre la mutabilité du système, selon lequel il s'agit d'un mécanisme complexe dont les locuteurs sont en grande partie inconscients, ne saurait être retenu, car les sujets ne sont pas si ignorants du système dont ils font un usage journalier: "on peut modifier certaines lois aussi inconsciemment qu'on les emploie et qu'on les maintient». Saussure, en posant l'incompétence des locuteurs devant la transformation de leur langue, suit la tradition néo-grammairienne qui réduit la langue à sa fonction purement intellectuelle, alors que l'histoire atteste que des ingérences sont possibles.

Il y a donc selon Jakobson quatre dualités successives qui divisent le langage, chacune débouchant sur une nouvelle subdivision : "Tout langage se présente simultanément sous deux aspects, langue et parole. Toute parole ainsi que toute langue comprend simultanément un aspect subjectif ou intersubjectif, et toute parole ainsi que toute langue, dans son aspect subjectif ainsi qu'intersubjectif relève simultanément de chacune des deux forces en présence, conformisme et non-conformisme dans leur aspect temporel ainsi que spatial ».

Ce texte révèle, de la part de Jakobson, une lecture très attentive du texte du CLG, jusqu'à l'intuition de ce sur quoi à l'époque il ne pouvait rien savoir de précis, le rôle des éditeurs ${ }^{10}$. Pour lui, le $C L G$, avec ses illuminations géniales et ses contradictions, constitue une étape entre la réflexion des Néo-grammairiens et celle du Cercle de Prague... et la sienne propre; une étape inachevée, à laquelle manquerait l'idée de fonction. On ne peut que regretter que la partie sur le signe qui suivait n'existe qu'à l'état d'esquisse. 


\section{Jakobson ultérieurement}

28

Jakobson saura prendre acte de l'incidence des recherches de Godel et de Engler, et il caractérisera Saussure « comme le grand douteur qui, toujours, voyait les deux aspects de chaque problème " (cité par Engler, 1990, qui renvoie à un propos de 1972) ${ }^{11}$. Dans le même temps, son intérêt s'élargit, au-delà des Sources, aux Anagrammes ${ }^{12}$.

Pourtant, à partir de son arrivée aux Etats-Unis, ses références ne sont plus tout à fait les mêmes, et la distance à l'égard du Saussure du CLG va s'accentuant, jusqu'à la conclusion apportée au tome II des Selected Writings («Retrospect», in 1971b, p. 711 sq), dont la différence de ton avec la conférence du Collège de France, pourtant à peu près contemporaine, reste surprenante. Ce «Retrospect» ne peut se lire autrement que comme, à l'automne d'une vie, un bilan définitif sur un thème. Or il s'y montre très critique vis-à-vis de Saussure, et, sur la plupart des points, beaucoup moins nuancé que dans ce que nous venons de voir.

Près de la moitié $d u$ «Retrospect » discute des thèses saussuriennes, dont il commence par mettre en cause l'originalité même: «la plupart des notions cardinales et des principes introduits par Saussure peuvent être attribués à ses aînés Baudouin de Courtenay et Kruszewski»; Saussure n'aurait que l'avantage d'une "manière plus explicite et ingénieuse » de présenter les faits. Les antinomies sont intéressantes, mais tant qu'elles demeurent « sans solution », « l'intégrité et l'unité de la linguistique étaient menacées »; « les progrès vers une synthèse des 'dualités internes' marquent vraiment l'étape post-saussurienne de la linguistique ». Et il n'est en fin de compte pas un concept du CLG qui soit approuvé sans des réserves plus ou moins décisives: langue/parole est reformulé en "code/message", et "la séparation absolue de ces deux aspects s'est transformée en la prise en compte de deux hiérarchies ", l'arbitraire du signe est mis en cause par l'iconicité, la linéarité du signifiant est selon lui remise en question par l'analyse des phonèmes en traits distinctifs, la non-pertinence de la substance est interrogée, une hiérarchie est introduite parmi les termes du paradigme, le signe est interprété dans les termes de la conception stoïcienne, le système est corrigé par la fonction («le système fonctionnel» de la première Thèse), et la synchronie est reformulée en synchronie dynamique ${ }^{13}$.

Mais alors, pourquoi Jakobson reste-t-il attaché à Saussure ?

Il tient à l'idée sémiologique de "langue comme système où tout se tient " (jamais « système de signes »). Mais l'aspect conceptuel de cette sémiologie n'est pas reconnu, la structure devient un postulat de référence, et non comme chez Saussure, quelque chose à construire. Ils n'est dès lors pas besoin de ce qui chez Saussure permet cette construction, la définition de l'objet au moyen des dichotomies. On peut donc déconstruire les concepts un à un, et l'idée même de "synthèse des dualités internes » remet en cause la nécessité de construire un objet.

Jakobson a pourtant trouvé chez Saussure quelque chose qui lui est essentiel, le principe de différence comme accès au système, dont il a fait, tant que le besoin s'en est pour lui fait sentir, une machine de guerre contre l'historicisme des Néo-grammairiens. Saussure est utilisé, sans que son originalité soit reconnue de façon globale, et sans que la lettre du texte soit toujours travaillée; ce que Cornélius van Schooneveld ${ }^{14}$ résume en la formule : «Il fallait bien un pavillon pour couvrir le navire ». 


\section{BIBLIOGRAPHIE}

Le Cercle de Prague, numéro spécial de Change, Paris, Seuil.

Engler R., 1990, « La parte di Saussure », in Roman Jakobson, a cura di Pietro Montani e Massimo Prampolini, Roma, Editori Riuniti.

Fontaine J., 1974, Le Cercle linguistique de Prague, Paris, Mame.

Holenstein E., 1984, Das Erbe Hegels, Frankfurt, Suhrkamp.

Jakobson R.O. 1971a, Selected Writings I, La Haye, Mouton.

Jakobson R.O. 1971b, Selected Writings II, La Haye, Mouton.

Jakobson R.O., 1973, Questions de poétique, Paris, Seuil.

Jakobson R.O., 1975, N.S. Trubetzkoy's Letters and Notes, The Hague-Paris, Mouton.

Jakobson R.O., 1984, « La théorie saussurienne en rétrospection », édité par L. Waugh, Linguistics $\mathrm{n}^{\circ} 22$.

Jakobson R., Karcevsky S., Troubetzkoy Prince N., 1928, «Quelles sont les méthodes les mieux appropriées à un exposé complet et pratique de la phonologie d'une langue quelconque ? ", in Actes du Ier Congrès International des Linguistes, Leiden, A.W. Sijthoff's Uitgeversmaatschappij N.V.

Rudy S., 1990, Roman Jakobson, a Complete Bibliography of his Writings, Berlin-New York, Mouton de Gruyter.

Troubetzkoy N.S., 1933, « La phonologie actuelle », Journal de psychologie, numéro exceptionnel de janvier, republié in Essais sur le langage, Paris, Ed. de Minuit, 1969.

Van Schooneveld C., 1992, interview par Françoise Gadet, 11 Août.

Viel M., 1984, La Notion de « marque » chez Trubetzkoy et Jakobson, Paris, Didier-Erudition.

Waugh L., 1984, « Introduction à Roman Jakobson : la théorie saussurienne », Linguistics n² 22.

\section{NOTES}

1. Sans accorder aux chiffres une importance autre qu'indicative (surtout que ceux-ci ne tiennent compte que de la mention du nom), précisons que le premier tome des Selected Writings («Phonological Studies») comporte, selon l'index des noms, 45 mentions de Saussure, le seul auteur davantage cité étant Troubetzkoy, avec 79 mentions ; quant au deuxième tome (« Word and Language »), il en comporte 65, très loin devant le deuxième auteur qui est Sapir, avec 37 mentions.

Il faut néanmoins préciser que Jakobson a souvent modifié légèrement ses propres articles au moment de les faire figurer dans les Selected Writings, et l'un des points de modification est justement la référence à Saussure, qu'il amplifie (cf. Viel 1984) : stratégie pour se faire lui-même entendre?

2. Dans une lettre de mai 1932, il écrit qu'il vient de le lire pour la seconde fois.

3. Dans la notice nécrologique qu'il consacre à Karcevski, Jakobson écrit: « He was the first who in 1917-1919, during his shortlived return to Russia, fired the young generation of Moscow 
linguists with the Cours de linguistique générale and applied its precepts to the study of contemporary Russian» («Sergej Karcevskij, August 281884 - November 71955 », in 1971b, p. $517 \mathrm{sq}$ ). Cette mention ne suffit pas toutefois pour reconstituer ce qu'a réellement été la réception à Moscou.

4. Le texte s'ouvre avec une référence à Saussure. Mais la traduction en français (reproduite in 1973) ne comporte pas ce passage, qu'on ne trouve que dans l'original en russe (Selected Writings V, p. 299 sq) et dans la tradution allemande, la seule qui soit complète.

En 1919, Jakobson ne peut pas avoir lu le CLG, et fait usage de termes transmis oralement par Karcevski, qui ne sont pas conservés dans le CLG, comme "poussière linguistique " (Viel, 1984, p. 58). Pour la période du Cercle de Prague, voir aussi Fontaine 1974.

5. Viel (1984, p. 63) suggère une piste interprétative de ce terme fréquent chez Jakobson, en remarquant que «dépasser» et "dépassement» alternent avec les termes allemands " aufheben » et " Aufhebung ». Mais cela suffit-il pour lire ici un effet de l'héritage hégélien, par ailleurs incontestable chez Jakobson (voir Holenstein, 1984)?

6. Il n'y a sur ce point aucun doute. La formulation même des signatures indique où git le problème: "Roman Jakobson, Prague; S. Karcevsky, Professeur adjoint à l’Université de Genève ; Prince N. Troubetzkoy, Professeur à l'Université de Vienne ». En 1927, Jakobson n'a pas encore de poste en Tchécoslovaquie. Voir Fontaine, 1974.

7. «La thèse de F. de Saussure définissant la langue comme un système de valeurs relatives est presque généralement admise dans la linguistique contemporaine » : telle est la première phrase de la Proposition qui est rédigée en français, comme d'ailleurs la question elle-même.

8. Année à la fois de la mort de Troubetzkoy et du Congrès de phonétique de Gand, où Jakobson présente le premier exposé développé de sa théorie des traits.

9. Deux lettres de Jakobson publiées dans le volume de Change intitulé «Le Cercle de Prague » signalent les parts prises, outre par lui-même, par Mukarovsky, Troubetzkoy, Mathesius, Havranek et Durnovo, avant même les effets d'une discussion générale au Cercle.

10. «Les éditeurs du Cours ont méconnu cette idée purement dialectique dans la doctrine de leur maître et par crainte qu'on ne reproche à Saussure 'd'être illogique ou paradoxal en attribuant à la langue deux qualités contradictoires', ils ont à tort essayé de présenter l'opposition des concepts antinomiques comme une simple façon d'expression ».

Van Schooneveld (1992) affirme que «Jakobson said even before the appearance of Godel's book that the Cours consisted of class notes taken by students and that a number of contradictions in the text had to be due to their misunderstanding ".

11. A cette époque, les marques d'un regain d'intérêt de Jakobson pour Saussure sont nombreuses.

Dans son discours de clôture du Neuvième Congrès International des Linguistes (1962, in 1971b), il évoque Saussure et l'importance du travail de Godel (p. 601); mis à part Humboldt, tous les autres linguistes mentionnés sont vivants et ont donné des communications au Congrès.

Par ailleurs, Rudolf Engler m'a aimablement communiqué les copies des lettres que Jakobson lui a écrites (de 1964 à 1975) à propos de la publication de son Edition Critique, qui montrent un intérêt immédiat et soutenu.

La citation reproduite par Engler (1990) provient du compte-rendu que Franck Marchand a présenté dans l'Education, où il cite des extraits des quatre conférences que Jakobson a données à Paris au Collège de France; l'une d'entre elles portait sur synchronie/diachronie.

12. Jakobson a contribué à la connaissance que l'on a de la genèse de ce travail, avec l'article « La première lettre de Ferdinand de Saussure à Antoine Meillet sur les anagrammes » (1971, reproduit in 1973). 
13. L'étude de chacun de ces points mérite d'être effectuée en détail, car ils ne manifestent pas tous le même degré de pertinence ou de finesse dans la lecture du CLG. Il resterait aussi à établir si la « synthèse des dualités internes » relève encore d'une inspiration hégélienne.

14. Van Schooneveld (1992) rapportait ainsi des propos de Jakobson lui-même, à qui il avait naguère adressé une question semblable à la mienne. 